\title{
Emergency Extracorporeal Membrane Oxygenation (ECMO)-Supported Percutaneous Coronary Interventions in the Fibrillating Heart
}

\author{
Mark J. Ricciardi, ${ }^{1}$ MD, Mauro Moscucci, ${ }^{1}$ MD, Bradley P. Knight, ${ }^{1}$ MD, Adam Zivin, ${ }^{1}$ MD, \\ Robert H. Bartlett, ${ }^{2}$ MD, and Eric R. Bates, ${ }^{1} *$ MD
}

\begin{abstract}
We describe two cases of refractory ventricular fibrillation complicating transcatheter interventional procedures. Extracorporeal membrane oxygenation was used in each to support percutaneous coronary revascularization in the fibrillating heart as a means of facilitating successful restoration of sinus rhythm. Cathet. Cardiovasc. Intervent. 48:402405, 1999. @ 1999 Wiley-Liss, Inc.
\end{abstract}

Key words: cardiopulmonary resuscitation; cardiopulmonary support; coronary angioplasty; ventricular fibrillation

\section{INTRODUCTION}

The use of cardiopulmonary bypass has allowed the surgical treatment of congenital and acquired diseases of the heart since 1954 [1]. Recently, portable cardiopulmonary support (PCPB or CPS) has been used prophylactically to support high-risk percutaneous coronary interventions and in angioplasty emergencies complicated by cardiac failure [2-4]. The hematologic sequelae of conventional CPS (hemolysis; loss of circulating volume, red cells, and clotting factors; and the frequent need for blood factor transfusion) typically restrict its use to less than $6 \mathrm{hr}$.

Extracorporeal membrane oxygenation (ECMO) is a form of cardiopulmonary bypass that couples the use of cardiopulmonary bypass machinery (mechanical pump, oxygenator, heat exchanger, arterial and venous cannulae, and tubing like that of CPS) with protocols and staff devoted to the prolonged use of extracorporeal circulatory support. Originally used in newborns and children with severe cardiac and respiratory failure $[5,6]$ and later for adult respiratory distress syndrome (ARDS) [7], it is classically viewed as a bridge to definitive therapy or disease resolution. The use of anticoagulation, fluid, and hemodynamic management protocols by a dedicated staff allows for the safe use of ECMO for weeks in the ICU. This makes it an ideal support system for patients expected to have prolonged recovery. While never tested as a support strategy for high-risk percutaneous interventions, the prolonged use of emergently instituted ECMO for primary cardiac failure in the adult (both spontaneous and in the setting of cardiac surgery) has been described with varying degrees of success [8-10].
We describe two patients with refractory ventricular fibrillation (VF), one complicating elective left anterior descending (LAD) artery angioplasty and the other complicating ventricular tachycardia (VT) catheter ablation. Both required aggressive resuscitation, circulatory support, and percutaneous revascularization in the fibrillating heart before successful defibrillation could be achieved.

\section{CASE REPORTS}

\section{Case 1}

A 54-year-old man, who 9 years previously had undergone coronary artery bypass grafting and carotid endarterectomy, presented with worsening angina pectoris and right lower extremity claudication. Pharmacologic stress testing and peripheral and coronary angiography showed impaired flow reserve in the anterior wall, severe narrowing of the right internal iliac artery, left ventricular ejection fraction of $40 \%$, severe three-vessel coronary artery disease, patent vein grafts to the right coronary and obtuse marginal arteries, and an occluded vein graft to the LAD. The native LAD, with significant disease at the

${ }^{1}$ Division of Cardiology, University of Michigan Medical Center, Ann Arbor, Michigan

2Division of General Surgery, University of Michigan Medical Center, Ann Arbor, Michigan

\footnotetext{
*Correspondence to: Dr. Eric R. Bates, Cardiac Catheterization Laboratory, University of Michigan Medical Center, UH B1 F245, 1500 East Medical Center Drive, Ann Arbor, MI 48109.
}

Received 17 February 1999; Revision accepted 25 June 1999 
ostium and mid segments, was thought to be responsible for his angina and appeared amenable to percutaneous revascularization.

The decision was made to proceed with percutaneous coronary angioplasty $(3 \times 15 \mathrm{~mm}$ NC Bandit, Scimed Life Systems, Maple Grove, MN) of the ostial and mid LAD lesions. This resulted in an improved but suboptimal lumen with no evidence of dissection. Soon thereafter, the patient developed ventricular fibrillation unresponsive to removal of the guiding catheter from the left coronary artery and immediate external defibrillation. Aggressive external cardiac compression was performed and endotracheal intubation established. Repeat angiography showed ostial LAD occlusion with loss of wire position from the distal artery. Resuscitative efforts were resumed. Multiple attempts at external defibrillation with 360 Joules using a standard monophasic defibrillator and an anteroposterior patch configuration proved ineffective despite intravenous loading with lidocaine, bretyllium, and amiodarone. Defibrillation was then successfully performed by adding a second external monophasic defibrillator with a sternum-to-apex configuration, and delivering 720 Joules by simultaneous manual delivery. Transient postconversion asystole was treated with right ventricular pacing. Despite multiple successful defibrillations using 720 Joules, ventricular fibrillation repeatedly recurred and sinus rhythm could not be maintained. While prolonged CPR and advanced cardiac life support measures maintained excellent perfusion pressure and oxygenation, severe acidemia developed and only brief episodes of organized ventricular tachycardia could be achieved with high-energy shocks. The decision was made to place femoral arterial and venous cannulae (21 and $23 \mathrm{Fr}$, respectively) for ECMO support.

The patient was successfully placed on circulatory support after 45 min of CPR, but the electrical storm continued unabated despite continued efforts and improvements in hemodynamics and acid-base disturbance. Attention was then redirected to revascularization, approximately $1 \mathrm{hr}$ after commencing cardiopulmonary resuscitation. While the patient remained in ventricular fibrillation, the proximal and mid LAD segments were successfully dilated and stented with three $3 \times 15 \mathrm{~mm}$ Palmaz-Schatz stents (Johnson and Johnson Interventional Systems, Warren, NJ). Thrombolysis in myocardial infarction (TIMI) grade 3 flow was restored and no residual stenosis was evident in the stented areas. On the first attempt after revascularization, the patient was successfully defibrillated with 720 Joules and returned to sinus rhythm.

He was transferred to the coronary care unit and supported with IV amiodarone, mechanical ventilation, and ECMO. Intact brain function was evident from the time of initial resuscitative efforts and the patient was responding to commands by the time of his arrival in the CCU. Surface echocardiography initially showed "stunning" of the anterior wall with gradual improvement in systolic function over the ensuing days. Before distal perfusion could be established, the left leg became ischemic and necrotic, requiring above-the-knee amputation. He was weaned from amiodarone and ECMO support within 5 days. He suffered no further myocardial ischemia or ventricular arrhythmias. A prolonged hospital course was complicated by pneumonia and finally necrotizing pancreatitis, which developed 30 days postangioplasty. The patient died during his second exploratory laparotomy.

\section{Case 2}

A 60-year-old man with a history of ischemic cardiomyopathy (ejection fraction 25\%), successful stenting of the mid right coronary artery (RCA) 4 months previously, heart failure, ventricular tachycardia (VT), implantable cardioverter defibrillator (ICD) therapy, and recent nonischemic adenosine thallium scan presented for VT ablation because of frequent ICD discharges. During the ablation procedure, he developed intractable VF refractory to multiple attempts at pharmacologic and electrical defibrillation. Aggressive CPR was performed and ECMO was instituted within $30 \mathrm{~min}$ using $23 \mathrm{Fr}$ right femoral vein and $21 \mathrm{Fr}$ right femoral artery cannulae. VF persisted and the patient underwent emergency coronary angiography, which showed $20 \%$ left main disease, subtotal narrowing of the proximal and mid LAD, 70\% and 90\% stenoses of the first obtuse marginal artery, occlusion of the second obtuse marginal artery, and interval occlusion of the mid RCA at the site of the previous stent implantation. There were left-to-right and right-to-right collaterals.

Heparin and abciximab (ReoPro, Centocor BV, the Netherlands) were given and the RCA occlusion was crossed and dilated (3.0-mm Bandit), resulting in a $10 \%$ residual diameter stenosis. The first obtuse marginal artery was also successfully dilated with the same balloon catheter. Immediately thereafter, the patient was successfully defibrillated with one 360-Joule shock.

With the preservation of brain, lung, and kidney but continued poor cardiac function over the ensuing 3 days, the patient was thought to be a suitable cardiac assist device and heart transplant candidate. Accordingly, left and right ventricular assist devices (HeartMate, ThermoCardiosystems, Woburn, MA) were implanted and ECMO discontinued. The patient was successfully extubated after 10 days, was later weaned from right ventricular support, remained on left ventricular support for 6 months, and then underwent successful orthotopic cardiac transplantation. He is alive 9 months later. 


\section{DISCUSSION}

While percutaneous coronary interventions and invasive electrophysiologic procedures are occasionally complicated by ventricular fibrillation [11-13], these events are rarely unresponsive to rapid electrical defibrillation. Previously reported is the use of emergency cardiopulmonary support to establish a more favorable milieu for successful defibrillation (i.e., improved oxygenation, perfusion, and acid-base balance). In previous reports of emergency bypass support [3,14-17], all patients with VF were successfully converted to sinus rhythm with the institution of CPS but prior to revascularization. The patients described herein represent situations in which extracorporeal support (and resultant corrections in hemodynamic and acid-base disturbance) alone was insufficient for reestablishing sinus rhythm and dictated the need for addressing the presumed ischemic etiology for the refractory electrical storm. These two cases therefore represent the first reports of percutaneous coronary interventions in the fibrillating heart made possible by the emergent institution of ECMO support. In case 1, efforts at recanalization of the occluded artery could not be achieved without prolonged periods of pulselessness and brain anoxia. In case 2, it was assumed that only relief of the ischemic burden would make defibrillation possible. Given the options of intra-aortic balloon pumping, CPS, or ECMO as "bailout" support measures, the latter was chosen because of an anticipated need for prolonged circulatory support (i.e., longer than the usual 6-hr limitation with CPS). At our institution, ECMO support can be achieved within minutes, especially in the cardiac catheterization laboratory where arterial and venous access is either already established or easily attained. Once placed "on circuit," patients are constantly monitored by a team of physicians, nurses, and technicians dedicated to the care of ECMO patients. This team accounts for the ability to institute rapidly and continue support for prolonged periods of time (sometimes up to 30 to 40 days).

Survival in the two patients described, despite prolonged ventricular fibrillation, stemmed from very effective initial external cardiac massage efforts, which allowed the operators time to define the inciting problem and begin circulatory support. In the first case, loss of wire position during CPR precluded immediate recanalization of the culprit vessel and dictated the need to stabilize the patient by extracorporeal means. Multiple attempts at defibrillation were unsuccessful in part because of an ischemic substrate and severe, uncorrectable acidemia. In the second case, ECMO support allowed time to transport the patient to the catheterization laboratory where revascularization could be performed. In both cases, maintenance of sinus rhythm was achieved only after successful revascularization was performed with the heart still fibrillating.

The ECMO techniques, contrasted to those of CPS, that contributed to the success in these patients were: heparin anticoagulation by continuous infusion, titrated to maintain whole blood activated clotting time (ACT) at $180 \pm 10 \mathrm{sec}$; venous drainage by siphon, rather than suction, to eliminate hemolysis; the exclusion of reservoirs or stagnant areas within the circuit; platelet transfusion to maintain platelet count greater than $100,000 / \mathrm{mm}^{3}$; and a dedicated team of caregivers using protocols designed for extended continuous perfusion in the ICU.

Our report demonstrates the first successful use of extracorporeal membrane oxygenation-supported percutaneous coronary revascularization in the fibrillating heart. Successful revascularization facilitated restoration of sinus rhythm in what was otherwise refractory ventricular fibrillation and represents dramatic evidence for an ischemic substrate in intractable ventricular fibrillation.

\section{REFERENCES}

1. Gibbon JH Jr. Application of a mechanical heart and lung apparatus to cardiac surgery. Minn Med 1954;37:171.

2. Phillips SJ, Zeff RH, Kongtohworn C, Skinner JR, Toon RS, Grignon A, Wickemeyer W, Iannone LA. Percutaneous cardiopulmonary bypass: application and indication for use. Ann Thorac Surg 1989;47:121-123.

3. Shawl FA, Domanski MJ, Wish MH, Davis M, Punja S, Hernandez TJ. Emergency cardiopulmonary bypass support in patients with cardiac arrest in the catheterization laboratory. Cathet Cardiovasc Diagn 1990;19:8-12.

4. Grambow DW, Deeb GM, Pavlides GS, Margulis A, O’Neill WW, Bates ER. Emergent percutaneous cardiopulmonary bypass in patients having cardiovascular collapse in the cardiac catheterization laboratory. Am J Cardiol 1994;73:872-875.

5. Bartlett RH, Gazzaniga AB, Jeffries MR, Huxtable RF, Haiduc NJ, Fong SW. Extracorporeal membrane oxygenation (ECMO) cardiopulmonary support in infancy. Trans Am Soc Artif Intern Organs 1976;22:80-93.

6. Bartlett RH, Andrews AF, Toomasian JM, Haiduc NJ, Gassaniga AB. Extracorporeal membrane oxygenation for newborn respiratory failure: forty-five cases. Surgery 1982;92:425-433.

7. Anderson H III, Steimle C, Shapiro M, Delius R, Chapman R, Hirschl R, Bartlett R. Extracorporeal life support for adult cardiorespiratory failure. Surgery 1993;114:161-172.

8. Pennington DG, McBride LR, Kanter KR, Miller LW, Ruzevich SA, Naunheim K, Swartz MT, Termuhlen D. Bridging to heart transplantation with circulatory support devices. J Heart Transplantation 1989;8:116-123

9. Delius RE, Zwischenberger JB, Cilley R, Behrendt DM, Bove EL, Deeb GM, Crowley D, Heidelberger KP, Bartlett RH. Prolonged extracorporeal life support of pediatric and adolescent cardiac transplant patients. Ann Thorac Surg 1990;50:791-795.

10. Reedy JE, Swartz MT, Raithel SC, Szukalski EA, Pennington DG. Mechanical cardiopulmonary support for refractory cardiogenic shock. Heart Lung 1990;19:514-523.

11. Dorros G, Cowley MJ, Simpson J, Bentivogilio LG, Block LG, Bourassa M, Detre K, Gosselin AJ, Kelsey SF, Kent KM, Mock MB, Mullin SM, Myler RK, Passamani ER, Stertzer SH, Williams 
DO. Percutaneous transluminal coronary angioplasty: report of complications from the National Heart, Ling and Blood Institute PTCA registry. Circulation 1983;67:723-730.

12. Steffenino G, Meier B, Finci L, Velebit V, von Segesser L, Faidutti B, Rutishauser W. Acute complications of elective coronary angioplasty: a review of 500 consecutive procedures. Br Heart J 1988;59:151-158.

13. Brendan E, Mahrer PR, Aharonian VJ. Incidence and presumed etiology of ventricular fibrillation during coronary angioplasty. Am J Cardiol 1991;67:769-770.

14. Shawl FA, Baxley WA. Role of percutaneous cardiopulmonary bypass and other support devices in interventional cardiology. Cardiol Clin 1994;12:543-557.
15. Raithel SC, Swartz MT, Braun PR, Dake SB, Taub JO, Zambie MA, Miller LW, Deligonul U, McBride LR, Pennington DG. Experience with an emergency resuscitation system. ASAIO Trans 1989;35:475-477.

16. Mori Y, Ueno K, Hattori A, Kim T, Aoyama T, Segawa T, Mimoto H, Tomita R, Tanaka T, Mori N. Emergency cardiopulmonary bypass support in patients with cardiac arrest caused by myocardial infarction. Artif Organs 1994;18:698-701.

17. Overlie PA, Walter PD, Hurd HP II, Wells GA, Seger JJ, Zias J, Wey RJ, Jensen JB, Shoukfeh MF, Levine MJ, et al. Emergency cardiopulmonary support with circulatory support devices. Cardiology 1994;84:231-237. 